\title{
Advances in Hydrological Forecasting
}

\author{
Minxue $\mathrm{He}^{1, *}$ and Haksu Lee ${ }^{2}$ \\ 1 California Department of Water Resources, 1416 9th Street, Sacramento, CA 95814, USA \\ 2 Len Technologies, 12139 Westwood Hills Dr, Oak Hill, VA 20171, USA; haksulee@hanmail.net \\ * Correspondence: minxuehe@gmail.com
}

Citation: He, M.; Lee, H. Advances in Hydrological Forecasting.

Forecasting 2021, 3, 517-519.

https: / / doi.org/10.3390/

forecast 3030032

Received: 6 July 2021

Accepted: 7 July 2021

Published: 8 July 2021

Publisher's Note: MDPI stays neutral with regard to jurisdictional claims in published maps and institutional affiliations.

Copyright: (c) 2021 by the authors. Licensee MDPI, Basel, Switzerland. This article is an open access article distributed under the terms and conditions of the Creative Commons Attribution (CC BY) license (https:// creativecommons.org/licenses/by/ $4.0 /)$.
Hydrological forecasting is of primary importance to better inform decision-making on flood management, drought mitigation, water system operations, water resources planning, and hydropower generation, among others. Typical hydrological forecasting translates single deterministic or an ensemble of short, intermediate, and long lead-time meteorological forecasts into estimates of hydrological variables of interest (e.g., streamflow, river stage, snowmelt, etc.) via forecast models at the corresponding temporal scales. These models range from process-based hydrological models to purely data-driven models. The model predictive skill and uncertainty are normally verified by comparing archived forecasts to field data or in a hindcasting mode. During forecasting, real-time in situ or remote sensing measurements for forecast hydrological variables can be assimilated into the forecast model to update model states or parameters for improved forecasts. Before being disseminated for operational use, hydrological forecasts are often post-processed to best reflect the perceptions of forecasters on the future state of those forecast variables.

Although there has been immense progress in forecasting systems, services, and observational sensors to date, hydrological forecasting today faces convoluted challenges induced by the increasing trend of extreme events, changing basin climate and hydrology, and demands of a unified and versatile hydrological forecasting system operating at local to continental scales. This Special Issue aimed to explore the latest methodological advances and novel applications in hydrological forecasting that tackle one or more of those challenges. The collection of papers published in this Special Issue covered a range of topics on improving hydrological forecasting via new datasets and innovative approaches. Each of these papers is summarized as follows.

$\mathrm{Wu}$ [1] proposed an approach using the bivariate meta-Gaussian distribution that allows the explicit treatment of precipitation intermittency, as well as a wide choice of parametric and non-parametric models for marginal distributions. $\mathrm{Wu}$ [1] presented the new proof for an intermittency equation of the model, and studied how to tune the bivariate meta-Gaussian distribution for an optimal fit. The two variables used are predicted singlevalued precipitation amounts and the corresponding observed precipitation amounts. Numerical simulations are implemented with data from the Global Ensemble Forecast System and four River Forecast Centers in the United States. The Mallows distance for the entire joint distribution is used to tune the parameter of the meta-Gaussian distribution. The optimization results were comparatively evaluated with results from using the sample correlation coefficient and maximum likelihood estimate as parameter values. The results suggested that tuning the dependence parameter has limited effects toward a better overall model fit, and that the goodness-of-fit of the conditional distribution can be improved in the case of optimizing the parameter for a targeted conditional distribution.

Bhuiyan et al. [2] explored the application of innovative machine-learning (ML)-based error models in improving the quality of satellite-based precipitation products. Specifically, the study applied two ML techniques including the Random Forecast (RF) and Neural Network (NN) to correct errors in the Integrated Multi-satellite Retrievals for Global Precipitation Measurement (IMERG) precipitation product. The proposed error models 
were exemplified in the Brahmaputra River Basin during a four-year period. The quantilequantile plot, along with a set of statistical metrics, were utilized to assess the performance of the error models. The findings indicated that both RF-based and NN-based error models lead to reduction in the random and systematic errors for all precipitation percentile ranges. The error corrected IMERG precipitation products showed a slightly higher improvement by the NN-based error model compared to the RF-based model. Overall, the NN-based error model slightly outperformed the RF-based error model. The authors expected that the ML-based error modeling algorithm is potentially applicable to ungauged areas or to the global scale, as well as for other types of remote-sensing precipitation products.

Pokhrel et al. [3] presented a framework to assess future flood rates and risks. The framework consists of a statistical analysis procedure, as well as a hydraulic modeling and risk assessment procedure. The former first bias-corrects streamflow projections based on the latest multi-model ensemble Coupled Model Inter-comparison Project Phase 6 (CMIP6) climate data. Future peak flows with 100-year and 500-year return periods are subsequently determined. The latter employs the one-dimensional HEC-RAS model to generate floodplain maps corresponding to 100-year and 500-year flood events. Next, four hazard categories (low, moderate, high, and severe) are produced for each extreme (100-year and 500-year) event. Different risk scores are also assigned to different land use types for vulnerability assessment. Lastly, the risk-maps for both existing and future extreme events are developed, and the risk assessment is conducted by comparing the present risk to the projected risk in the future. The framework was applied to a $32-\mathrm{km}$ long reach of the Neuse River in North Carolina, United States. The results indicated an increase in flood inundation area and thus higher flood hazards and risks in the future in the study area. The authors claimed that the findings of the study have the potential to inform policymakers in terms of preparing flood risk mitigation plans.

Lee et al. [4] developed a Mean Field Bias (MFB)-aware variational (VAR) assimilation framework, or MVAR, to account for catchment-wide biases in distributed hydrologic model states. MVAR corrects the MFB in model states first, and then updates the resulting states at the grid box scale. MVAR was comparatively evaluated with the conventional VAR based on results from assimilating streamflow into the distributed Sacramento Soil Moisture Accounting model for the $2258 \mathrm{~km}^{2}$ headwater basin draining into the Elk River near Tiff City, Missouri. Compared to the conventional VAR, MVAR reduced the mean squared error of streamflow by $2-8 \%$ at the outlet, and by $1-10 \%$ at the interior location. MVAR adjusts model states at remote cells from the outlet by larger margins than the VAR.

Giannaros et al. [5] introduced a novel WRF-Hydro-based fluvial flood forecasting system for the Southeast Mediterranean (SEM) and presented modeling methodologies and forecasting schemes used in the system. The initial prototype results derived under a preoperational mode were presented, and future developments and challenges were discussed.

In conclusion, this Special Issue presented the latest studies on methodological and operational advances in hydrological forecasting.

Funding: This research received no external funding.

Institutional Review Board Statement: Not applicable.

Informed Consent Statement: Not applicable.

Data Availability Statement: Not applicable.

Acknowledgments: We sincerely thank the authors who contributed to this Special Issue, as well as the reviewers who provided insightful comments to the authors.

Conflicts of Interest: The authors declare no conflict of interest. 


\section{References}

1. $\mathrm{Wu}, \mathrm{L}$. Tuning the Bivariate Meta-Gaussian Distribution Conditionally in Quantifying Precipitation Prediction Uncertainty. Forecasting 2020, 2, 1-19. [CrossRef]

2. Bhuiyan, M.A.E.; Yang, F.; Biswas, N.K.; Rahat, S.H.; Neelam, T.J. Machine Learning-Based Error Modeling to Improve GPM IMERG Precipitation Product over the Brahmaputra River Basin. Forecasting 2020, 2, 248-266. [CrossRef]

3. Pokhrel, I.; Kalra, A.; Rahaman, M.; Thakali, R. Forecasting of Future Flooding and Risk Assessment under CMIP6 Climate Projection in Neuse River, North Carolina. Forecasting 2020, 2, 323-346. [CrossRef]

4. Lee, H.; Shen, H.; Seo, D.-J. Mean Field Bias-Aware State Updating via Variational Assimilation of Streamflow into Distributed Hydrologic Models. Forecasting 2020, 2, 526-548. [CrossRef]

5. Giannaros, C.; Galanaki, E.; Kotroni, V.; Lagouvardos, K.; Oikonomou, C.; Haralambous, H.; Giannaros, T. Pre-Operational Application of a WRF-Hydro-Based Fluvial Flood Forecasting System in the Southeast Mediterranean. Forecasting 2021, 3, 437-446. [CrossRef] 